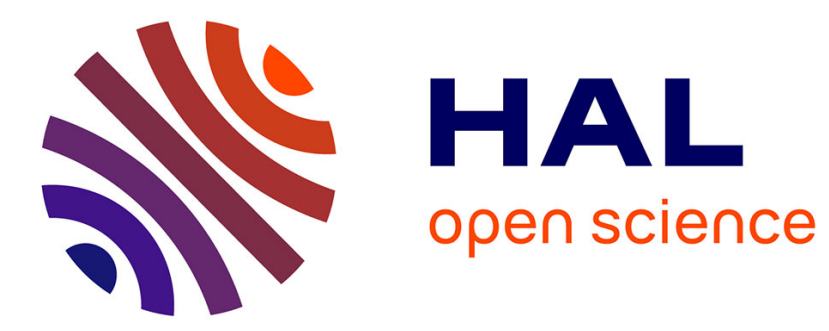

\title{
Theory of viscous buckling of multilayered fluids undergoing finite strain
}

Maurice A. Biot

\section{To cite this version:}

Maurice A. Biot. Theory of viscous buckling of multilayered fluids undergoing finite strain . Physics of Fluids, 1964, 7 (6), pp. 855-861. 10.1063/1.1711296 . hal-01368663

\section{HAL Id: hal-01368663 \\ https://hal.science/hal-01368663}

Submitted on 21 Sep 2016

HAL is a multi-disciplinary open access archive for the deposit and dissemination of scientific research documents, whether they are published or not. The documents may come from teaching and research institutions in France or abroad, or from public or private research centers.
L'archive ouverte pluridisciplinaire HAL, est destinée au dépôt et à la diffusion de documents scientifiques de niveau recherche, publiés ou non, émanant des établissements d'enseignement et de recherche français ou étrangers, des laboratoires publics ou privés. 


\section{AIP | Physics of Fluids}

Theory of Viscous Buckling of Multilayered Fluids Undergoing Finite Strain M. A. Biot

Citation: Physics of Fluids 7, 855 (1964); doi: 10.1063/1.1711296

View online: http://dx.doi.org/10.1063/1.1711296

View Table of Contents: http://scitation.aip.org/content/aip/journal/pof1/7/6?ver=pdfcov

Published by the AIP Publishing

\section{Articles you may be interested in}

Nonlinear hydrodynamic damping of sharp-edged cantilevers in viscous fluids undergoing multiharmonic base excitation

J. Appl. Phys. 112, 124908 (2012); 10.1063/1.4769307

Ship waves on a viscous fluid of finite depth

Phys. Fluids 9, 940 (1997); 10.1063/1.869189

Comments on "Viscous buckling of thin fluid layers"

Phys. Fluids 24, 1764 (1981); 10.1063/1.863602

Viscous buckling of thin fluid layers

Phys. Fluids 24, 1 (1981); 10.1063/1.863241

The Fundamental Problem of the Theory of Viscous Fluids

J. Appl. Phys. 14, 244 (1943); 10.1063/1.1714981

\section{Looking for a specific} instrument?

Easy access to the latest equipment. Shop the Physics Today Buyer's Guide.

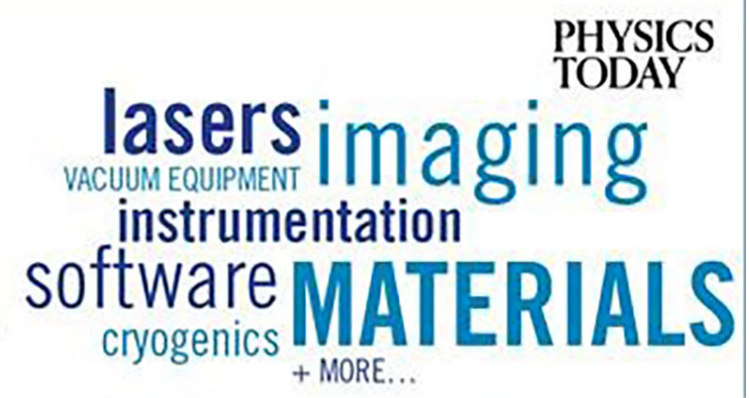




\title{
Theory of Viscous Buckling of Multilayered Fluids Undergoing Finite Strain
}

\author{
M. A. BioT \\ Shell Development Company, New York, New York
}

(Received 5 December 1963)

\begin{abstract}
The equations of fluid mechanics are applied to the problem of small perturbations upon a finite initial strain-rate of a viscous fluid. The magnitude of the viscosity is such that inertia forces are negligible. General solutions are developed for the time history of buckling of a fluid with an arbitrary number of layers of different viscosities under finite compressive deformation. The effect of gravity is taken into account. Numerical solutions are derived for the single layer. Results are compared with values obtained from the theory of elasticity and viscoelasticity. The interest of the theory lies in its application to problems of folding of geological structures.
\end{abstract}

\section{INTRODUCTION}

$\mathrm{T}$ HE theory of stability of multilayered continua has been developed by the writer in several papers. In particular, for an incompressible medium the problem was treated extensively for the embedded layer ${ }^{1,2}$ and for the system with an arbitrary number of layers. ${ }^{3,4}$ Slow motion is assumed so that inertia forces may be excluded. The theory is rigorously applicable to elastic and viscoelastic media initially at rest. Its application to a viscous fluid which is initially in a state of flow involves an approximation which is valid provided the total deformation remains small.

The purpose of the present paper is to develop a theory of stability for incompressible viscous fluids which is rigorously valid for a large compressive deformation. We assume, therefore, that a small perturbation is superposed upon an initial state of flow with arbitrary finite strain.

Sections 2 and 3 derive the time-dependent finite strain in a viscous fluid under uniform constant stress, and bring out the existence of an apparent instability of purely kinematic origin. In Secs. 4 and 5 it is shown how a perturbation field of a fluid plate initially in a state of flow may be evaluated by introducing fictitious tangential stresses at the boundary. The viscous buckling of an isolated plate as a function of time is evaluated numerically. In Sec. 6 these results are applied to formulate the general differential equations for the time history of buckling of a multilayered viscous fluid. The effect of gravity is included. The equations are applied to the case of a single layer embedded in an infinite medium. It is also pointed out that the equations are applicable in the general case where

\footnotetext{
1 M. A. Biot, Quart. J. Appl. Math. 27, 185 (1959).

${ }^{2}$ M. A. Biot and H. Ode, Quart. J. Appl. Math, 19, 351

${ }^{3}$ M. A. Biot, J. Franklin Inst. 276, 128 (1963).

4 M. A. Biot, J. Franklin Inst. 276, 231 (1963).
} (1962). the initial strain-rate is three-dimensional and timedependent.

While the mathematical form of the equations is fundamentally different from those derived for the corresponding theories of elasticity and viscoelasticity it is found that the numerical solutions become the same as those for the viscous fluid when the instability is of significant magnitude.

The interest of the present theory resides in its application to a large class of problems of tectonic folding of stratified geological structures.

\section{CONSTANT STRAIN-RATE IN A VISCOUS FLUID}

Consider the plane motion of an incompressible fluid of high viscosity $\eta$. We assume the motion to be slow so that the inertia forces are negligible. The velocity components $v_{x}$ and $v_{y}$ in the $(x, y)$ plane satisfy the Navier-Stokes equations.

$$
\eta \nabla^{2} v_{z}+\partial \sigma / \partial x=0, \quad \eta \nabla^{2} v_{y}+\partial \sigma / \partial y=0,
$$

where $\sigma$ is the negative pressure. Incompressibility is expressed by the equation

$$
\partial v_{x} / \partial x+\partial v_{y} / \partial y=0 .
$$

Assuming Newtonian viscosity the stress components $\sigma_{x x}, \sigma_{y y}$, and $\sigma_{x y}$ are related to the velocity gradients by the equations

$$
\begin{gathered}
\sigma_{x x}-\sigma=2 \eta \partial v_{x} / \partial x, \quad \sigma_{y y}-\sigma=2 \eta \partial v_{y} / \partial y \\
\sigma_{x y}=\eta\left(\partial v_{y} / \partial x+\partial v_{x} / \partial y\right)
\end{gathered}
$$

A state of uniform constant stress, $\sigma_{x x}=S_{11}$, $\sigma_{y y}=S_{22}, \sigma_{x y}=0$, produces constant strain-rates. Combining Eqs. (2.2) and (2.3) these strain-rates are found to be

$$
-\partial v_{x} / \partial x=\partial v_{y} / \partial y=p_{0}
$$

with

$$
p_{0}=P / 4 \eta, \quad P=S_{22}-S_{11} .
$$

Consider $x$ and $y$ to be the coordinates of a fluid 


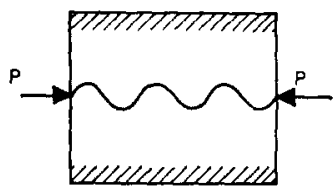

(a)

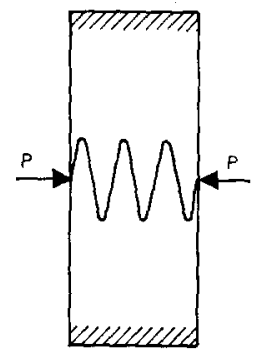

(b)
Fra. 1. Kinematic instability due to a uniform strain-rate.

particle and $\dot{x}, \dot{y}$ their time derivatives. Equations (2.4) may be written

$$
-\dot{x} / x=\dot{y} / y=p_{0} .
$$

Hence by integration

$$
x=x_{0} e^{-p_{0} t}, \quad y=y_{0} e^{p_{0} t},
$$

with $x_{0}, y_{0}$ representing the particle coordinate at $t=0$. Considering this flow from the viewpoint of finite strain we may define two extension ratios,

$$
\lambda_{1}=x / x_{0}=e^{-p_{0} t}, \quad \lambda_{2}=y / y_{0}=e^{p_{0} t} .
$$

The value $P$ of the stress is the "effective" compression. Without loss of generality we may put $S_{22}=0$. This is a consequence of the fact that we may always cancel $S_{22}$ by adding or subtracting an over-all hydrostatic pressure without altering the mechanics of the deformation.

\section{KINEMATIC INSTABILITY}

An interesting consequence of the foregoing result is an apparent instability which is of purely kinematic origin. Consider the case of a fluid undergoing a plane-strain deformation under a uniform compression $P=-S_{11}$. A rectangular region of this fluid at $t=0$ is shown in Fig. 1(a). We imagine a sinusoidal line to be drawn in the fluid along the direction of $P$. After a time $t$ the fluid has been squeezed in this direction and the corresponding compressive strain is measured by the extension ratio $\lambda_{1}=$ $\exp \left(-p_{0} t\right)$. In the direction normal to the compression the expansion measured by the extension ratio $\lambda_{2}=\exp \left(p_{0} t\right)$. The sinusoidal line has followed the motion of the fluid particles and is now compressed accordionwise in the direction of the compression and has been stretched in the normal direction as shown in Fig. 1(b). The stretching is proportional to the increasing exponential function of time $\lambda_{2}=\exp \left(p_{0} t\right)$ and exhibits the mathematical features of an instability.
Such instability is of course spurious and of purely kinematic nature. It requires a considerable amount of compressive strain before it becomes significant.

\section{BOUNDARY CONDITION}

Consider again a plane strain deformation under the constant compression $P$. Let us consider the wavy line in Fig. 1 as a boundary where the fluid medium lies in the region below this line. In order to maintain an undisturbed steady flow we must apply tangential and normal stresses at the boundary as shown in Fig. 2. The $x$ axis is oriented in the same direction at the compression $P$. The ordinate of the boundary is denoted by $u_{y}$ and its slope $\alpha=\partial u_{y} / \partial x$ is assumed to be small. Hence the boundary stresses are

$$
\sigma_{12}=P \partial u_{y} / \partial x, \quad \sigma_{22}=0 .
$$

Let us superimpose an instantaneous velocity field, of components $v_{x}$ and $v_{y}$ at the boundary. This produces additional boundary stresses $\bar{\sigma}_{12}$ and $\bar{\sigma}_{22}$. The total stresses are

$$
\sigma_{12}=\bar{\sigma}_{12}+P \partial u_{y} / \partial x, \quad \sigma_{22}=\bar{\sigma}_{22} .
$$

The important point about these additional stresses is that they are determined entirely by substituting the additional velocity field into the Navier-Stokes equations (2.1)-(2.3). This is a consequence of the fact that these equations are linear and therefore the stresses $\bar{\sigma}_{12}$ and $\bar{\sigma}_{22}$ may be evaluated as if the fluid were initially at rest and unstressed.

Consider the $x$ axis to represent a plane of fluid particles in the undisturbed steady state. We assume the disturbance to be sinusoidally distributed along $x$. The shape of the deformed boundary is

$$
u_{y}=V \cos l x
$$

and the boundary stresses are written

$$
\begin{array}{ll}
\sigma_{12}=\tau \sin l x, & \bar{\sigma}_{12}=\bar{\tau} \sin l x, \\
\sigma_{22}=q \cos l x, & \bar{\sigma}_{22}=\bar{q} \cos l x .
\end{array}
$$

Equations (4.2) become

$$
\tau=\bar{\tau}-P l V, \quad q=\bar{q} .
$$

Fig. 2. Normal and tangential stresses at the boundary.

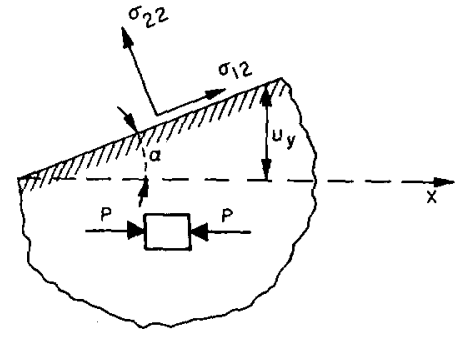


The velocity disturbances at the boundary are also distributed sinusoidally and written

$$
v_{x}=U^{\prime} \sin l x, \quad v_{y}=V^{\prime} \cos l x .
$$

We now use an important kinematic relation for the time derivative $\dot{u}_{\nu}$ of $u_{\nu}$. The quantity $\dot{u}_{v}$ represents the velocity of the fluid particle at the boundary measured relatively to the moving $x$ axis. This velocity is equal to

$$
\dot{u}_{y}=v_{y}+p_{0} u_{y} \text {. }
$$

The term $p_{0} u_{y}$ represents the additional velocity due to the initial strain rate (2.6). By substituting into Eq. (4.7) the sinusoidal distributions (4.3) and (4.6), we obtain

$$
V^{\prime}=\dot{V}-p_{0} V .
$$

\section{VISCOUS BUCKLING OF A FLUID PLATE}

We now apply the foregoing results to a viscous fluid plate undergoing a plane-strain deformation under a compressive stress $P$ acting along the axis of the plate. The initial thickness of the plate at the time $t=0$ is $h_{0}$. (Fig. 3). At the time $t$ the thickness has become

$$
h=h_{0} e^{p_{0} t} .
$$

Consider the plate to have a slight initial waviness which is the same on top and bottom and is sinusoidally distributed along $x$. This corresponds to a plate deformation of the flexural type, hence antisymmetric with respect to its axis. The initial wavelength of this deformation is $L_{0}$. At time $t$ the wavelength has become

$$
L=L_{0} e^{-p_{0} t} .
$$

The deformation of the upper surface of the plate is represented by the sinusoidal distribution (4.3) of the ordinate $u_{\nu}$ of the surface with

$$
l=2 \pi / L \text {. }
$$

Both $V$ and $l$ are functions of time. The $x$ axis is chosen to coincide with the moving but undeformed plane boundary of the plate in the initial state of steady flow.

The surface in this case is assumed to be free. Hence the boundary condition is $\tau=q=0$. With these values Eqs. (4.5) become

$$
\bar{\tau}=P l V, \quad \bar{q}=0 .
$$

The stresses $\bar{\tau}$ and $\bar{q}$ are the stresses due to the perturbation velocities at the top boundary. These velocities are sinusoidally distributed and expressed by Eqs. (4.6). The values of $U^{\prime}$ and $V^{\prime}$ are completely
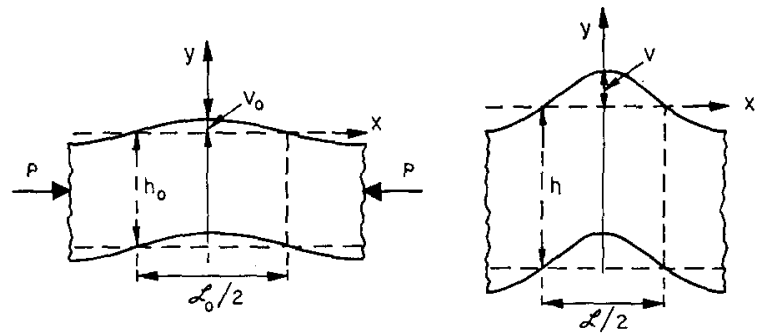

Fig. 3. Buckling of a viscous fluid plate.

determined by the surface stresses $\bar{\tau}$ and $\bar{q}$. They are obtained by solving the Navier-Stokes equations (2.1) for a fluid plate of thickness $h$ initially at rest and with the boundary stresses given by (5.4).

This problem is the same as for an elastic plate of incompressible isotropic material free of initial stress. The displacements are replaced by the velocities and the elastic modulus by the viscosity coefficient. The problem was solved in an earlier paper. ${ }^{3}$ For antisymmetric deformation the solution is written

$\bar{\tau} / \eta l=a_{11} U^{\prime}+a_{12} V^{\prime}, \quad \bar{q} / \eta l=a_{12} U^{\prime}+a_{22} V^{\prime}$.

The coefficients are

$$
\begin{gathered}
a_{11}=\frac{4 \cosh ^{2} \gamma}{\sinh 2 \gamma+2 \gamma}, \quad a_{12}=-\frac{4 \gamma}{\sinh 2 \gamma+2 \gamma}, \\
a_{22}=\frac{4 \sinh ^{2} \gamma}{\sinh 2 \gamma+2 \gamma},
\end{gathered}
$$

with a nondimensional wavelength parameter

$$
\gamma=\frac{1}{2} l h=\pi h / L .
$$

According to Eqs. (5.2) and (5.3) it is a function of time. We write

$$
\gamma=\kappa e^{2 p_{0} t}, \quad \kappa=\pi h_{0} / L_{0} .
$$

By introducing the values (5.4) and substituting $P / \eta=4 p_{0}$, Eqs. (5.5) become

$a_{11} U^{\prime}+a_{12} V^{\prime}=4 p_{0} V, \quad a_{12} U^{\prime}+a_{22} V^{\prime}=0$.

We eliminate $U^{\prime}$ from these two equations and substitute the value (4.8) for $V^{\prime}$. This yields the ordinary differential equation

$$
\dot{V} / V=4 \gamma p_{0} /(\sinh 2 \gamma-2 \gamma)+p_{0} .
$$

Since $\gamma$ is a function of time, the integral is obtained by quadrature. We write

$$
V / V_{0}=A(t) e^{p_{0} t}
$$

with

$$
\log A=\int_{0}^{t} \frac{4 \gamma p_{0}}{\sinh 2 \gamma-2 \gamma} d t
$$




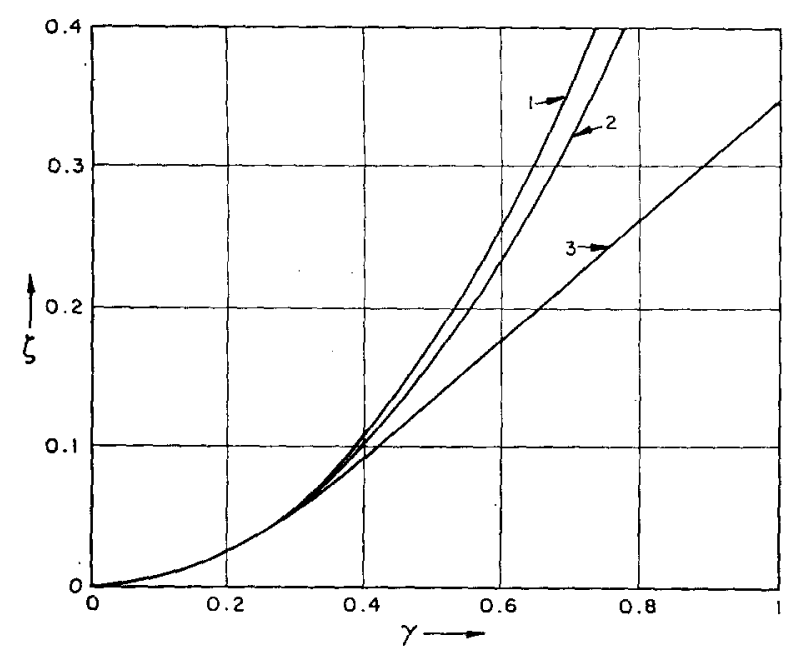

FIg. 4. Stability parameter for a plate derived from (1) viscous fluid theory, (2) thin elastic plate theory, and (3) the theory of elasticity.

The initial value of $V$ at $t=0$ is denoted by $V_{0}$ [see Fig. 3].

The amplification factor $A(t)$ represents an intrinsic instability. It is superposed on the kinematic instability represented by the factor $\exp \left(p_{0} t\right)$. For the purpose of comparison with other theories it is useful to put

$$
A(t)=\exp \left(\int_{0}^{t} p d t\right)
$$

We may then write

$$
\zeta=\frac{2 p_{0}}{p}=\frac{P}{2 \eta p}=\frac{1}{2 \gamma}(\sinh 2 \gamma-2 \gamma) .
$$

The quantity $p$ represents an instantaneous rate of amplification of the plate deflection.

For wavelengths which are sufficiently large in comparison with the thickness the value of $\gamma$ is small and we may write approximately

$$
\zeta=\frac{2}{3} \gamma^{2} .
$$

This value is the same as obtained from the Euler theory of buckling of a thin elastic plate by replacing the elastic modulus by the corresponding operator $\eta p .^{1,4}$

The exact value (5.14) of $\zeta$ is plotted as curve 1 in Fig. 4. The approximate value (5.15) is plotted as curve 2. We have also plotted the value of $\zeta$ derived in an earlier paper ${ }^{4}$ from the exact theory of stability of an elastic continuum under initial stress and applying the principle of viscoelastic correspondence (curve 3).

It is seen that all three values are practically identical for $\gamma<0.3$ hence for wavelengths larger than about ten times the thickness.

The intrinsic amplification $A(t)$ may be obtained by evaluating the integral (5.12). Since $d \gamma=2 p_{0} \gamma d t$ we may use $\gamma$ as variable of integration. We derive

$$
\log A=\frac{3}{4 \kappa} F(2 \kappa)-\frac{3}{4 \gamma^{2}} F(2 \gamma)
$$

with

$$
\begin{gathered}
F(x)=\frac{1}{3} x^{2} \int_{x}^{\infty} \frac{d \xi}{\sinh \xi-\xi}, \\
\kappa=\pi h_{0} / L_{0} .
\end{gathered}
$$

The parameter $\kappa$ is the initial value of $\gamma$. Numerical values of the function $F(x)$ are given in Table I.

TABLE I. Values of $F(x)$ from Eq. (5.17).

\begin{tabular}{llll}
\hline \multicolumn{1}{c}{$x$} & $F(x)$ & $x$ & $F(x)$ \\
\hline 0.10 & $\mathbf{0 . 9 9 6 0}$ & 1.0 & 0.8297 \\
0.2 & $\mathbf{0 . 9 8 6 8}$ & 1.2 & 0.7802 \\
0.3 & 0.9739 & 1.4 & 0.7297 \\
0.4 & 0.9582 & 1.6 & 0.6793 \\
0.5 & 0.9403 & 1.8 & 0.6295 \\
0.6 & 0.9206 & 2.0 & 0.5810 \\
0.7 & 0.8994 & 3.0 & 0.3694 \\
0.8 & 0.8770 & 4.0 & 0.2200 \\
0.9 & 0.8537 & 8.0 & 0.0410 \\
\hline
\end{tabular}

\section{Intrinsic Surface Stability of a Viscous Fluid}

The foregoing results may be applied to a viscous plate of infinite thickness. In this limiting case the problem becomes one of surface stability of a viscous half-space. This case is obtained by putting $\gamma=\infty$ in Eq. (5.12). We derive

$$
\log A=0 .
$$

Hence when inertia and body forces are neglected there is no intrinsic first order instability of the surface of a viscous fluid. The amplification in this case is reduced to the factor $\exp \left(p_{0} t\right)$, which represents a purely kinematic effect.

This is in contrast with the case of an elastic medium or a medium approximately at rest initially and purely viscous for incremental deformations. In the latter case, which resembles plastic behavior, it was shown by the writer that surface instability is present ${ }^{1}$ and will cause surface wrinkling.

\section{VISCOUS BUCKLING OF A MULTILAYERED FLUID}

We first consider an isolated layer of thickness $h$. We again assume a deformation sinusoidally dis- 
tributed along the axis but this time it is not restricted to the case where it is antisymmetric across the thickness. Hence both flexure and variation in thickness of the layer are taken into account.

We must consider values at both top and bottom surface of the plate for the applied stresses $\tau$ and $q$, the perturbation stresses $\bar{\tau}$ and $\bar{q}$, the perturbation velocities $U^{\prime}$ and $V^{\prime}$ and the normal displacement $V$. We attach a subscript 1 to the variables at the top face and a subscript 2 for the variables at the bottom face.

The four perturbation stresses $\bar{\tau}_{1}, \bar{q}_{1}, \bar{\tau}_{2}$, and $\bar{q}_{2}$ are due entirely to the perturbation velocities $U_{1}^{\prime}, V_{1}^{\prime}$, $U_{2}^{\prime}$, and $V_{2}^{\prime}$ at the two faces. The relation between these quantities has been derived in a previous paper in the context of the theory of elasticity. ${ }^{3}$ It may be immediately extended to the case of a fluid by replacing the elastic modulus by the viscosity coefficient and the displacements by velocities. We must introduce the six coefficients

$$
\begin{array}{ll}
A=\frac{1}{2}\left(a_{11}+b_{11}\right), & D=\frac{1}{2}\left(a_{11}-b_{11}\right), \\
B=\frac{1}{2}\left(a_{12}+b_{12}\right), & E=\frac{1}{2}\left(a_{12}-b_{12}\right), \\
C=\frac{1}{2}\left(a_{22}+b_{22}\right), & F=\frac{1}{2}\left(a_{22}-b_{22}\right) .
\end{array}
$$

The values of $a_{11}, a_{12}$, and $a_{22}$ were already given by Eqs. (5.6). The other three coefficients were derived earlier. ${ }^{3}$ They are

$$
\begin{gathered}
b_{11}=\frac{4 \sinh ^{2} \gamma}{\sinh 2 \gamma-2 \gamma}, \quad b_{12}=\frac{4 \gamma}{\sinh 2 \gamma-2 \gamma}, \\
b_{22}=\frac{4 \cosh ^{2} \gamma}{\sinh 2 \gamma-2 \gamma} .
\end{gathered}
$$

We also introduce a quadratic function $I$ of the four perturbation velocities. We write

$$
\begin{aligned}
I & =\frac{1}{2} A\left(U_{1}^{\prime 2}+U_{2}^{\prime 2}\right)-D U_{1}^{\prime} U_{2}^{\prime} \\
& +\frac{1}{2} C\left(V_{1}^{\prime 2}+V_{2}^{\prime 2}\right)+F V_{1}^{\prime} V_{2}^{\prime} \\
& +B\left(U_{1}^{\prime} V_{1}^{\prime}-U_{2}^{\prime} V_{2}^{\prime}\right)+E\left(U_{1}^{\prime} V_{2}^{\prime}-U_{2}^{\prime} V_{1}^{\prime}\right)
\end{aligned}
$$

Relations between perturbation stresses and velocities may then be expressed in very compact form, as shown in some previous papers. ${ }^{3,4}$ These relations are

$$
\begin{array}{ll}
\bar{\tau}_{1}=\ln \frac{\partial I}{\partial U_{1}^{\prime}}, & \bar{\tau}_{2}=-\operatorname{l\eta } \frac{\partial I}{\partial U_{2}^{\prime}}, \\
\bar{q}_{1}=\ln \frac{\partial I}{\partial V_{1}^{\prime}}, & \bar{q}_{2}=-\ln \frac{\partial I}{\partial V_{2}^{\prime}} .
\end{array}
$$

From Eq. (4.5) the applied stresses are

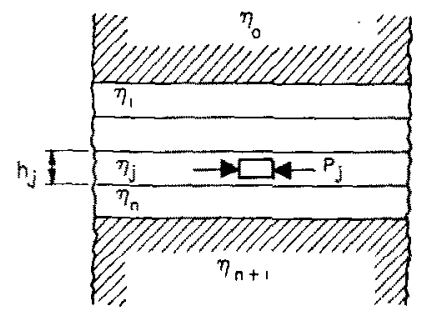

Fra. 5. System of $n$ fluid layers with initial compressive stress $P_{j}$.

$$
\begin{array}{ll}
\tau_{1}=\operatorname{l\eta } \frac{\partial I}{\partial U_{1}^{\prime}}-P l V_{1}, & \tau_{2}=-\ln \frac{\partial I}{\partial U_{2}^{\prime}}-P l V_{2} \\
q_{2}=\operatorname{l\eta } \frac{\partial I}{\partial V_{1}^{\prime}}, & q_{2}=-\operatorname{l\eta } \frac{\partial I}{\partial V_{2}^{\prime}} .
\end{array}
$$

Consider now a system of $n$ layers (Fig. 5). The layer numbered $j$ has a thickness $h_{j}$, a viscosity $\eta_{i}$, and an initial stress $P_{i}$. We denote by the subscript $j$ the values of velocities and stresses at the top face of this layer and by the subscript $j+1$ the values at the bottom face. The quadratic function $I_{i}$ attached to the layer $j$ is obtained from Eq. (6.3) by writing subscripts $j$ and $j+1$ instead of 1 and 2 , and replacing $\gamma$ by $\gamma_{j}=\frac{1}{2} l h_{i}$ in the values of the coefficients. We now equate the stresses $\tau$ and $q$ in two adjacent layers $j$ and $j+1$ at an interface. We find

$$
\begin{gathered}
\frac{\partial}{\partial U_{i+1}^{\prime}}\left(\eta_{i} I_{i}+\eta_{i+1} I_{i+1}\right)=\left(P_{i+1}-P_{i}\right) V_{i}, \\
\frac{\partial}{\partial V_{i+1}^{\prime}}\left(\eta_{i} I_{j}+\eta_{i+1} I_{i+1}\right)=0 .
\end{gathered}
$$

If the layers are embedded between two viscous half-spaces we consider them as layers of infinite thickness denoted by the subscripts 0 and $n+1$. Putting $\gamma=\infty$ the quadratic functions representing the half-spaces are drastically simplified to

$I_{0}=2\left(U_{1}^{\prime 2}+V_{1}^{\prime 2}\right), \quad I_{n+1}=2\left(U_{n+1}^{\prime 2}+V_{n+1}^{\prime 2}\right)$

Still more compact expression for the equation are obtained by using the total quadratic form

$$
g=\sum_{i=0}^{n+1} \eta_{j} I_{j}
$$

Equations (6.6) are then written

$\partial \mathscr{g} / \partial U_{i+1}^{\prime}=\left(P_{j+1}-P_{j}\right) V_{j}, \quad \partial \mathscr{S} / \partial V_{i+1}^{\prime}=0$.

From Eq. (4.8) the value of $V_{j}^{\prime}$ is

$$
V_{i}^{\prime}=\dot{V}_{i}-p_{0} V_{i} \text {. }
$$

By substituting these values into Eqs. (6.9) they become a system of $2 n+2$ linear differential 
equations with the time derivatives $\dot{V}_{i}$ for the $2 n+2$ unknown $U_{;}^{\prime}$ and $V_{i}$ at the $n+1$ interfaces.

The case where the stack of layers is free at one end or both is derived by putting either $\eta_{0}=0$, or $\eta_{n+1}=0$, or both, in the equations.

Note that by Eq. (2.5) we may also write

$$
P_{i+1}-P_{i}=4 p_{0}\left(\eta_{i+1}-\eta_{i}\right) \text {. }
$$

Integration of the linear system (6.9) yields the time history of viscous buckling of the multilayered fluid. The coefficients of the equations are those of the quadratic functions $I_{i}$. They are functions of time through the variables

$$
\gamma_{i}=\frac{1}{2} l h_{i}=\kappa_{i} e^{2 p_{0} t},
$$

where $\kappa_{i}$ represents the value of $\frac{1}{2} l h_{i}$ at the time $t=0$.

The effect of gravity on the stability may be added by using exactly the same method as in the case of elastic media which amounts to using an analog model, replacing the effect of gravity by interfacial buoyancy forces. ${ }^{4}$ If we include these gravity forces, Eqs. (6.9) become

$\frac{\partial \mathscr{g}}{\partial U_{i+1}^{\prime}}=\left(P_{j+1}-P_{i}\right) V_{i}, \frac{\partial \mathscr{g}}{\partial V_{j+1}^{\prime}}+\frac{\partial \mathcal{G}}{\partial V_{i+1}}=0$,

where

$$
\mathcal{S}=\frac{1}{2 l} \sum_{i=0}^{n}\left(\rho_{i+1}-\rho_{i}\right) g V_{i+1}^{2}
$$

$\rho_{i}$ is the density of $j$ th layer, and $g$ is the acceleration of gravity. Further simplification of these equations is obtained by introducing variables $\dot{\mathfrak{u}}_{j} \mathfrak{v}_{i}$ through the relations

$$
V_{i}=\mathfrak{v}_{j} e^{\mathbf{p}_{0} t}, \quad U_{i}^{\prime}=\dot{\mathfrak{u}}_{j} e^{p_{0} t} .
$$

We derive

$$
V_{i}^{\prime}=\dot{V}_{i}-p_{0} V_{i}=\dot{\mathrm{v}}_{i} e^{p_{0} t}
$$

By substituting these values in equations (6.13) the factor $\exp \left(p_{0} t\right)$ cancels out. They become

$$
\frac{\partial \mathcal{G}^{\prime}}{\partial \dot{\mathfrak{u}}_{i+1}}=\left(P_{i+1}-P_{i}\right) \mathfrak{v}_{i}, \quad \frac{\partial \mathcal{G}^{\prime}}{\partial \dot{\mathfrak{v}}_{i+1}}=-\frac{\partial \mathcal{G}^{\prime}}{\partial \mathfrak{y}_{i+1}} .
$$

The quadratic form $g^{\prime}$ is obtained from $g$ by replacing $U_{j}^{\prime}$ and $V_{j}^{\prime}$ by $\dot{\mathfrak{u}}_{i}$ and $\dot{\mathfrak{v}}_{i}$. Similarly $\mathcal{G}^{\prime}$ is obtained from $\mathcal{G}$ by substituting $\mathfrak{v}_{i}$ instead of $V_{i}$. The new variables $\mathfrak{u}_{i}$ and $\mathfrak{v}_{i}$ represent the intrinsic instability since the factor $\exp \left(p_{0} t\right)$ corresponding to the kinematic effect has been eliminated.

\section{Analog Model}

Attention is called to the physical significance of Eq. (6.17). They correspond to an analog model where the interfacial velocities $\dot{\mathfrak{u}}_{i}$ and $\dot{\mathfrak{v}}_{i}$ are the same as for a fluid at rest in a given instantaneous configuration under the action of tangential and vertical forces represented by the right side of Eqs. (6.17).

\section{Single Embedded Layer}

As an example consider a single layer of thickness $h$ viscosity $\eta$ and stress $P$ embedded between two fluid half-spaces of viscosity $\eta_{1}$ and stress $P_{1}$, (Fig. 6).

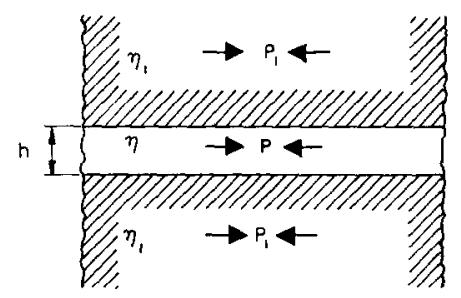

Fia. 6. Layer embedded in an infinite medium.

The buckling is of the flexural type. Gravity forces are neglected. Using Eqs. (4.5) with the values (5.5) for $\bar{\tau}$ and $\bar{q}$ we derive for the stresses at the top face of the layer

$$
\begin{aligned}
& \tau=\eta l\left(a_{11} U^{\prime}+a_{12} V^{\prime}\right)-P l V, \\
& q=\eta l\left(a_{12} U^{\prime}+a_{22} V^{\prime}\right) .
\end{aligned}
$$

On the other hand the stresses in the upper half-space at the interface are obtained by considering the bottom surface of a layer of infinite thickness. This amounts to putting $\gamma=\infty$ and changing the sign of $q$ and $U^{\prime}$ in Eq. (6.18). We derive

$\tau=-2 \eta_{1} l U^{\prime}-P_{1} l V, \quad q=-2 \eta_{1} l V^{\prime}$.

Equating the values (6.18) and (6.19) for the stresses at the interface we find

$$
\begin{gathered}
\left(\eta a_{11}+2 \eta_{1}\right) U^{\prime}+\eta a_{12} V^{\prime}+\left(P_{1}-P\right) V=0, \\
\eta a_{12} U^{\prime}+\left(\eta a_{22}+2 \eta_{1}\right) V^{\prime}=0 .
\end{gathered}
$$

As in Eq. (6.15) we put

$$
V=\mathfrak{b} e^{p_{0} t}, \quad U^{\prime}=\mathfrak{i t} e^{p_{0} t}
$$

Substitution in Eq. (6.20) yields

$$
\begin{gathered}
\left(\eta a_{11}+2 \eta_{1}\right) \dot{\mathfrak{u}}+\eta a_{12} \dot{\mathfrak{v}}+\left(P_{1}-P\right) \mathfrak{v}=0, \\
\eta a_{12} \dot{\mathfrak{u}}+\left(\eta a_{22}+2 \eta_{1}\right) \dot{\mathfrak{v}}=0 .
\end{gathered}
$$

By eliminating $\mathfrak{u}$ we derive the value of the intrinsic 
amplification factor $A$. We write the result in the form

$$
\zeta=P / 2 \eta p \quad p=\dot{\mathfrak{v}} / \mathfrak{v}=\dot{A} / A
$$

The stability parameter is

$$
\begin{aligned}
\zeta=\frac{1}{2 \gamma(1-n)} & {\left[\left(1+n^{2}\right) \sinh 2 \gamma\right.} \\
& \left.+2 n \cosh 2 \gamma+\left(n^{2}-1\right) 2 \gamma\right] .
\end{aligned}
$$

It is a function of $\gamma=\frac{1}{2} l h$ and of the ratio of viscosities

$$
n=P_{1} / P=\eta_{1} / \eta_{2} .
$$

For large viscosity contrasts and large wavelengths $(n \ll 1, \gamma \ll 1)$ we find the approximate value

$$
\zeta=\frac{2}{3} \gamma^{2}+n / \gamma
$$

This is the same as the value derived by applying the Euler theory of buckling of thin elastic plates neglecting interfacial friction and replacing the elastic moduli $\mu$ and $\mu_{1}$ by the corresponding operators $\eta p$ and $\eta_{1} p$. $^{1,5}$

The value of $\gamma$ for which $\zeta$ is a minumum defines the "dominant" or buckling wavelength. It is defined as the wavelength whose amplitude increases at the fastest rate. ${ }^{5}$

For large compressive strain we derive

$$
\log A=\int_{0}^{t} p d t=\int_{x}^{\gamma} \frac{d \gamma}{\gamma \zeta} .
$$

This expression yields the intrinsic amplification $A$ as a function of time.

\footnotetext{
5 M. A. Biot, Proc. Roy. Soc. (London) A242, 444 (1957).
}

\section{Generalization to Three-Dimensional and Variable Initial Strain-Rate}

In the foregoing analysis it was assumed for the sake of simplicity that the initial strain-rate is constant and two-dimensional. However the results may be readily extended to more general conditions provided we retain the assumption that the perturbation remains two-dimensional.

For three-dimensional initial strain-rate in the $j$ th layer we write

$$
P_{i}=S_{22}-S_{11}^{(i)}=2 \eta_{i}\left(p_{0}+p_{0}^{\prime}\right),
$$

where the finite strains are

$\lambda_{1}=\exp \left[-\int_{0}^{t} p_{0}^{\prime}(t) d t\right], \quad \lambda_{2}=\exp \left[\int_{0}^{t} p_{0}(t) d t\right]$.

The strain-rates $p_{0}$ and $p_{0}^{\prime}$ may now be arbitrary functions of time. The value of $\gamma_{j}$ is

$$
\gamma_{i}=\kappa_{j} \lambda_{2} \lambda_{1}^{-1}=\kappa_{i} \exp \left[\int_{0}^{t}\left(p_{0}+p_{0}^{\prime}\right) d t\right]
$$

and expression (6.11) is replaced by

$$
P_{i+1}-P_{j}=2\left(\eta_{i+1}-\eta_{i}\right)\left(p_{0}+p_{0}^{\prime}\right),
$$

which is now a function of time.

With these definitions the differential equations retain the same form as for the case of two-dimensional and constant initial strain-rate.

In the absence of gravity forces, attention is called to the possibility of simplifying the differential equations by using $\lambda_{2} \lambda_{1}^{-1}$ as independent variable instead of the time. This was already illustrated by Eqs. (5.16) and (6.27). 